\title{
Meta-analysis of the prevalence of depression among breast cancer survivors in Iran: an urgent need for community supportive care programs
}

\author{
Hassan Ahmadi Gharaei ${ }^{1}$, Mostafa Dianatinasab ${ }^{2}$, Seyyed Mostafa Kouhestani ${ }^{3}$, \\ Mohammad Fararouei ${ }^{4}$, Hossein Moameri ${ }^{1}$, Reza Pakzad ${ }^{5,6}$, Reza Ghaiasvand ${ }^{7}$ \\ 'Department of Epidemiology, Faculty of Public Health, Tehran University of Medical Sciences, Tehran, Iran; ${ }^{2}$ Department of Epidemiology, \\ Center for Health Related Social and Behavioral Sciences Research, Shahroud University of Medical Sciences, Shahroud, Iran; ${ }^{3}$ Department \\ of Health Management and Economics, School of Public Health, Tehran University of Medical Sciences, Tehran, Iran; ${ }^{4}$ Department of \\ Epidemiology, Faculty of Public Health, Shiraz University of Medical Sciences, Shiraz, Iran; ${ }^{5}$ Noor Research Center for Ophthalmic Epidemiology, \\ Noor Eye Hospital, Tehran, Iran; ${ }^{6}$ Faculty of Health, Ilam University of Medical Sciences, Ilam, Iran; ${ }^{7}$ Oslo Centre for Biostatistics and \\ Epidemiology, Department of Biostatistics, Institute of Basic Medical Sciences, University of Oslo, Oslo, Norway
}

OBJECTIVES: Depression, which is the most common comorbidity in breast cancer (BC) patients, has adverse effects on patients' quality of life, disease progress, and survival.

METHODS: The protocol of this study was registered in PROSPERO (registration No. CRD42019121494). We electronically searched published studies through January 2019 with the aim of finding articles that investigated the prevalence of depression among BC survivors. Web of Science, Scopus, PubMed/MEDLINE, Science Direct, and Google Scholar were searched to obtain relevant published studies. This review included 14 cross-sectional and 4 cohort studies published from 2000 to 2018 . We used a random-effects model to conduct the meta-analysis and generated a summary estimate for the pooled prevalence with $95 \%$ confidence intervals (CIs). A subgroup analysis was also conducted based on the depression assessment tool used and the study design.

RESULTS: The total sample size of the studies contained 2,799 women with BC, including 1,228 women who were diagnosed with depression. The pooled prevalence of depression among Iranian women with BC was 46.83\% (95\% CI, 33.77 to 59.88) with significant heterogeneity $\left(\mathrm{I}^{2}=98.5 \%\right.$; $\left.\mathrm{p}<0.001\right)$. The prevalence of depression ranged from $14.00 \%$ (95\% CI, 4.91 to 23.09$)$ to $95.90 \%$ (95\% CI, 91.97 to 99.83 ). The results of the subgroup analyses suggested that the depression assessment tool, year of publication, and study design were sources of heterogeneity.

CONCLUSIONS: Our findings indicate a high prevalence of depression among BC patients, underscoring the urgent need for clinicians and health authorities to provide well-defined social and psychological supportive care programs for these patients.

KEY WORDS: Breast carcinoma, Depression, Supportive care, Quality of life, Prevalence, Meta-analysis

\author{
Correspondence: Mostafa Dianatinasab \\ Department of Epidemiology, Center for Health Related Social \\ and Behavioral Sciences Research, Shahroud University of Medical \\ Sciences, Shahroud 3614773955, Iran \\ E-mail: dianatinasab@sums.ac.ir \\ Received: May 15, 2019 / Accepted: Jul 4, 2019 / Published: Jul 4, 2019 \\ This article is available from: http://e-epih.org/ \\ (C) This is an open-access article distributed under the terms of the Creative \\ Commons Attribution License (http://creativecommons.org/licenses/by/4.0/), \\ which permits unrestricted use, distribution, and reproduction in any medium, \\ provided the original work is properly cited. \\ (C) 2019, Korean Society of Epidemiology
}

\section{INTRODUCTION}

It is estimated that about 2.1 million newly diagnosed cases of breast cancer (BC) occurred in 2018 worldwide, accounting for almost 1 in 4 of all cases of cancer among women. BC is the most frequently diagnosed cancer in most countries (154 of 185), and is the leading cause of death due to cancer in over 100 countries [1]. In Iran, BC is the most common cancer in women; it has a high mortality rate [2], and the mean age at diagnosis is significantly lower in Iranian women than in their Western counterparts $[3,4]$. As a result, it is among the most important challenges in 
public health [5]. However, because of newly advanced diagnostic and treatment strategies [6,7], the survival rate of patients has increased significantly. As a result, about $89 \%$ of patients now survive for at least 5 years after diagnosis [8]. Thus, focus has now turned toward enhancing patients' quality of life (QoL), as patients often experience persistent aversive symptoms such as fatigue, cognitive difficulties, and mental problems [9]. The increase in patients' life expectancy is accompanied by a longer exposure to negative psychological impacts of $\mathrm{BC}$ [9].

When diagnosed with cancer, patients are exposed to an instigator of many fears, including fears of death, separation, and isolation from their loved ones, as well as fear of deterioration and pain. The most common psychological disorders among cancer patients are mood disorders, anxiety, depression, and sexual dysfunction. For example, the period from the diagnosis of $\mathrm{BC}$ to months following primary therapy is long and highly stressful, which causes psychological instability and depression [5]. Depression, which is the most common comorbidities among BC survivors, diminishes QoL and may lead to an increase in the cost of healthcare [10]. An international study showed an increasing prevalence of depression among women with BC [11]. Therefore, promoting mental and physical health is becoming an important aspect of all related healthcare programs [6]. Evidence has suggested that depression may affect the progression and survival of cancer [12,13].

Helping patients with cancer achieve the best possible QoL is a shared goal among medical and healthcare communities [14]. Hence, with regard to the increasing number of $\mathrm{BC}$ patients and the increasing prevalence of psychological disorders among BC survivors, it is very important to understand the prevalence of depression and its impact on patients' health status. Robust findings on this topic underscore the urgent need for clinicians and health authorities to provide well-defined social and psychological supportive care programs for BC survivors.

\section{MATERIALS AND METHODS}

The present systematic review and meta-analysis was prepared according to the PRISMA (Preferred Reporting Items for Systematic Reviews and Meta-Analyses) statement [15].

\section{Protocol and registration}

The purpose of the present study was to determine the prevalence of depression among women with BC in Iran. The study protocol was registered in PROSPERO, an international prospective register of systematic reviews, under the registration No. CRD42019121494.

\section{Search strategy and selection criteria}

A comprehensive search was performed with the aim of finding any cross-sectional or cohort studies that investigated the prevalence of depression among patients with BC in Iran. Accordingly, the following sources were searched: Web of Science, Scopus, Pub-
Med/MEDLINE, Science Direct, Google Scholar, Magiran, Scientific Information Database, IranMedex, and Medlib. Four sets of related $\mathrm{MeSH}$ and non-MeSH terms in titles, abstracts, or keywords were used: (1) "breast cancer" OR "breast carcinoma" OR "breast neoplasm", (2) "depression" and (3) "prevalence" OR "occurrence" and (4) "Iran" were searched for articles published from February 2000 until January 2019.

The search strategy was performed using Boolean operators (AND, OR). Two authors independently reviewed the articles (HAG and SMK) and discrepancies were resolved by discussing with the third author (MD). We also manually reviewed the reference lists of related articles for other possibly relevant articles that were not found through the electronic search strategy.

\section{Inclusion criteria}

Prospective cohort studies and cross-sectional studies that investigated the prevalence of depression among Iranian BC patients were included, encompassing both studies on newly diagnosed $\mathrm{BC}$ cases (incident cases) and prevalent cases of BC.

\section{Exclusion criteria}

Prospective cohort studies and cross-sectional studies that did not report the prevalence of depression among Iranian BC patients, reported the prevalence of depression among patients with other types of cancer (not relevant or modified data), reported the prevalence of depression among other comorbidities of $\mathrm{BC}$ patients, or focused on BC recurrence were not included in our systematic review and meta-analysis. Studies in review design or letter to the editors (not a research article) were also excluded.

\section{Data extraction}

Two authors (HAG and SMK) independently extracted the following data for each study: authors' name, publication year, setting, sample size, numbers of patients who suffered from depression, prevalence of depression, age, the tool used, marital status, and study design. If the full text of any article was unavailable or if key information was missing from the reported data, up to 2 attempts to contact the authors were made at 1-week intervals, and we also sent an email to the publishers (e.g., the Elsevier and Wiley online library).

\section{Quality of assessment}

An 8-item checklist for the critical appraisal of studies of the prevalence/incidence of health problems [16] was used to examine the quality of eligible studies by 2 independent investigators (HAG and SMK). The checklist contained items assessing the following criteria: (1) whether a random sample or a whole population was used, (2) the use of an unbiased sampling frame, (3) adequacy of the sample size, (4) the use of standard measures, (5) whether outcome measurements were made by unbiased assessors, (6) adequacy of the response rate, (7) confidence intervals (CIs) and subgroup analyses, and (8) whether the study subjects were described. Each item was scored as 1 if a study met the crite- 
rion, and the scores were summed up. The range of the total score was from 0 (lowest possible quality) to 8 (highest possible quality). The quality assessment results were also checked by a third investigator (MD).

\section{Statistical analysis}

A random-effects model was used to investigate the pooled prevalence of depression with 95\% CIs among women with BC. The $\mathrm{I}^{2}$ test was used to evaluate the heterogeneity of studies [17]. Subgroup analyses were performed based on the study design, year of publication, the depression assessment tool used and quality of studies. To study the heterogeneity of sources, meta-regression was used based on the design and quality of studies, sample size, age of women, the tool used to assess depression, and publication year. The Egger test [18] and funnel plot method [19] were used to test the severity of publication bias. Moreover, a sensitivity analysis was conducted to assess the stability of the results. Stata version 14 (StataCorp., College Station, TX, USA) was used for the statistical analysis [20].

\section{Ethics statement}

Ethical approval was obtained from Tehran University of Medical Sciences. This was a review study, so consent to participate was unnecessary.

\section{RESULTS}

\section{Included studies}

As described in Figure 1, which shows the PRISMA flow chart, a total of 453 records were obtained by electronic and manual searching. After duplicate references were removed, 315 records remained for further assessment. We excluded 264 studies after screening titles and/or abstracts due to the following reasons: not being relevant $(n=207)$, not having been conducted in $\operatorname{Iran}(n=13)$, not having the full text available $(n=12)$, being a review/letter to the editor or poster presentation $(n=6)$, and not reporting the prevalence of depression $(n=26)$. Therefore, 51 studies remained to be carefully checked by examining the full texts, of which 33 articles were excluded for the following reasons: not containing relevant data $(n=17)$, not being an original article (i.e., being a re-

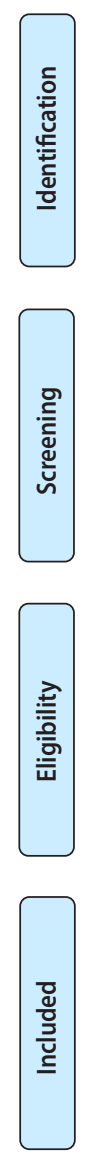

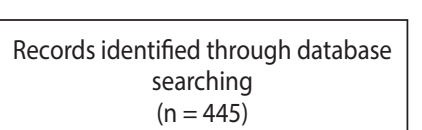

$(n=445)$

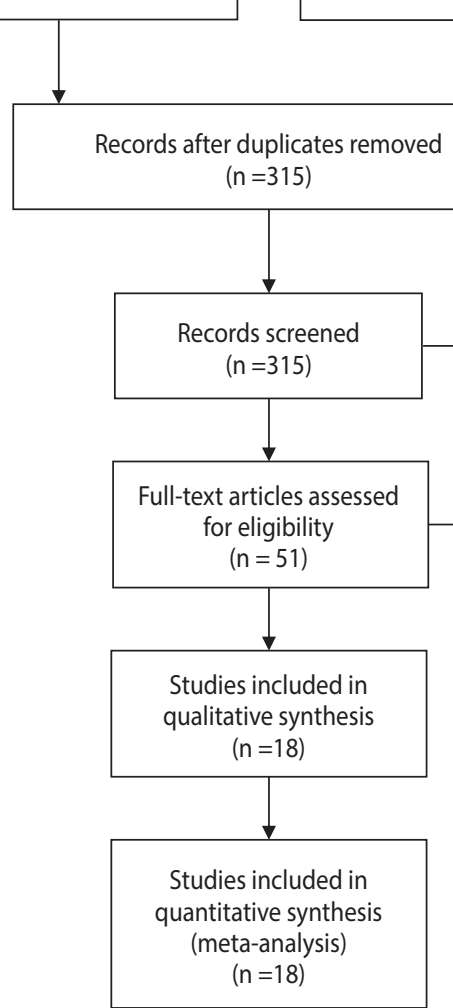

Additional records

(reference checking) $(n=8)$

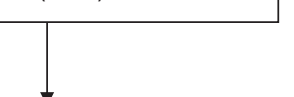




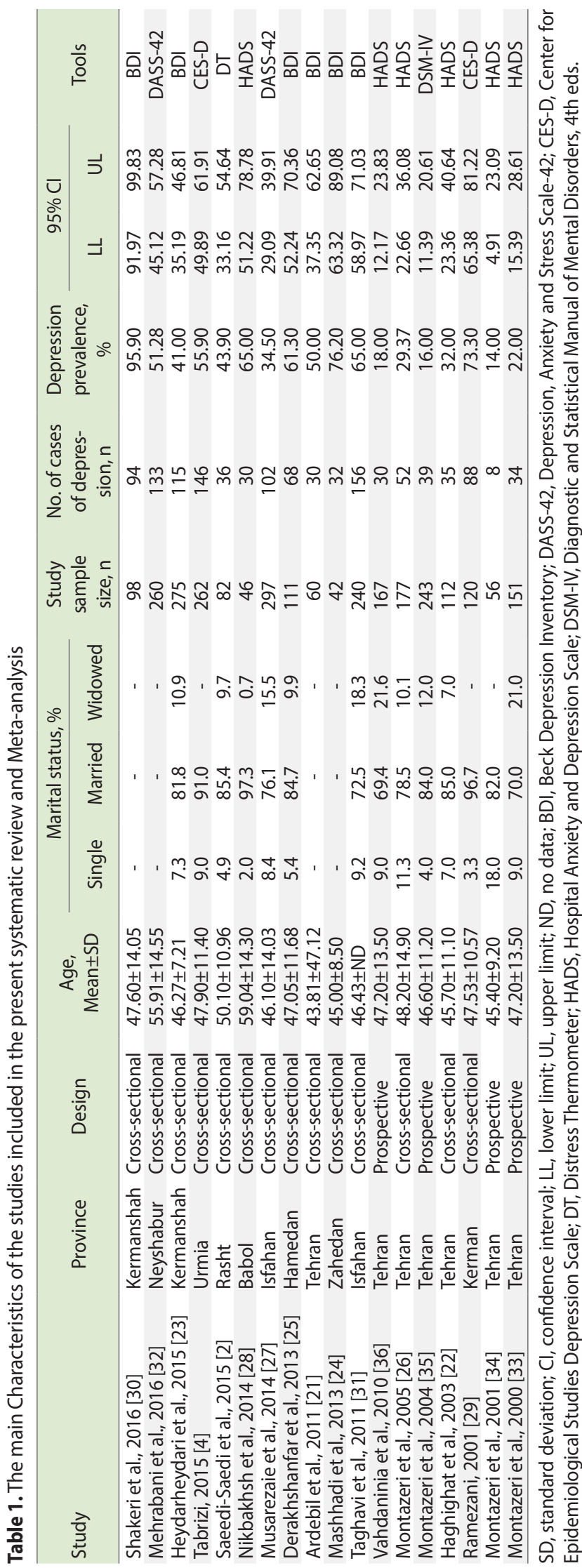

view article or letter to the editor, $n=5)$, using modified data $(n=7)$, or not reporting the outcome of interest $(n=4)$. In total, 18 articles met the criteria for qualitative synthesis in the systematic review and the quantitative meta-analysis. The characteristics of the studies included in this review are presented in Table 1 . The study design was cross-sectional $(n=14)[2,4,21-32]$ or cohort $(n=4)$ [33-36].

Overall, the included studies contained 2,799 women with histologically confirmed BC (mean sample size, $155.50 \pm 87.35$ patients). Of those patients, 1,228 (43.87\%) were diagnosed with depression, and the mean age of the patients was 47.94 years (standard deviation, 3.76).

Six different methods were used to diagnose depression in the included studies. Seven studies used the Beck Depression Inventory (BDI) [21,23-25,29-31], 2 studies used the Depression, Anxiety, and Stress Scale-42 (DASS-42) [27,32], 6 studies used the Hospital Anxiety and Depression Scale (HADS) [22,26,28,33,34, 36], 1 study used the Diagnostic and Statistical Manual of Mental Disorders, 4th edition (DSM-IV) [35], 1 study used the Distress Thermometer (DT) [2], and 1 study used the Center for Epidemiological Studies Depression Scale (CES-D) [4].

\section{Assessment of methodological quality}

According to the critical appraisal of studies of prevalence/incidence of a health problem checklist [16], 2 studies were classified as high-quality studies (score $\geq 7$ ) [33,36], 6 as medium-quality studies (score between 4 and 6) [21,22,24,28,30,35] and 10 as lowquality studies (score < 4) [2,4,23,25-27,29,30,32,34] (Supplementary Material 1).

\section{Pooled prevalence of depression}

The highest prevalence of depression among women with BC was $95.90 \%$ (95\% CI, 91.97 to 99.83 ) which was reported by Shakeri et al. [30] in Kermanshah, and the lowest prevalence was $14.00 \%$ (95\% CI, 4.91 to 23.09) reported by Montazeri et al. [34] in Tehran. Using a random-effects model, we found that the pooled prevalence of depression among women with BC was $46.83 \%$ (95\% CI, 33.77 to 59.88). The results of the included studies showed highly significant heterogeneity $\left(\mathrm{I}^{2}=98.5 \%\right.$; $\left.\mathrm{p}<0.001\right)$.

Table 2. Heterogeneity among the selected studies based on metaregression

\begin{tabular}{lrrrl}
\hline \multirow{2}{*}{ Variables } & \multirow{2}{*}{ Beta } & \multicolumn{2}{c}{$95 \% \mathrm{Cl}$} & \\
\cline { 3 - 4 } & & \multicolumn{1}{c}{$\mathrm{LL}$} & $\mathrm{UL}$ & p-value \\
Quality of study & 6.60 & -10.51 & 23.72 & 0.42 \\
Design & -37.72 & -58.47 & -16.97 & 0.001 \\
Age & 0.19 & -1.21 & 1.59 & 0.77 \\
Year & 9.42 & 4.28 & 2.20 & 0.04 \\
Sample size & -0.06 & -0.02 & 0.07 & 0.32 \\
Tools & -14.25 & -27.02 & -1.47 & 0.03 \\
\hline
\end{tabular}

$\mathrm{Cl}$, confidence interval; LL, lower limit; UL, upper limit. 


\section{Meta-regression}

Meta-regression was conducted according to study design, the measurement method, participants' age, the article's publication year, sample size, and studies' quality score (Table 2). Heterogeneity was found for study design, the measurement method, and the publication year of the article. Based on the results of meta-regression, the prevalence of depression had a significant association with study design $(\beta=-37.72 ; p=0.001)$. The prevalence of depression in the prospective studies was $37 \%$ lower than in crosssectional studies (Table 2). Furthermore, the prevalence of depression had a significant association with the measurement method $(\beta=-14.25 ; p=0.03)$ (Table 2$)$. The prevalence of depression measured by the BDI was $66.18 \%$ (95\% CI, 48.29 to 84.08 ), that measured by the HADS was $28.92 \%$ (95\% CI, 18.99 to 38.84 ) and that measured by other methods was $40.19 \%$ (95\% CI, 24.16 to 56.23 ) (Figure 2). We found a significant upward trend in the prevalence of depression among women with BC in recent years. As shown in Figures 3 and 4, the prevalence of depression during 2000-2004 was $31.41 \%$ (95\% CI, 11.29 to 51.53 ), during 2005-2009 it was $29.37 \%$ (95\% CI, 22.66 to 36.08 ), between 2010 and 2014 it was $52.48 \%$ (95\% CI, 35.82 to 69.14 ), and in 2015 or later it was $57.70 \%$ (95\% CI, 33.37 to 82.04$)(\beta=9.42 ; \mathrm{p}=0.04)$.

\section{Publication bias and sensitivity analysis}

The Egger test and funnel plot method were used to measure publication bias. As presented in Figure 5, no significant publication bias was found $(t=-0.57 ; \mathrm{p}=0.57)$. The sensitivity analysis is

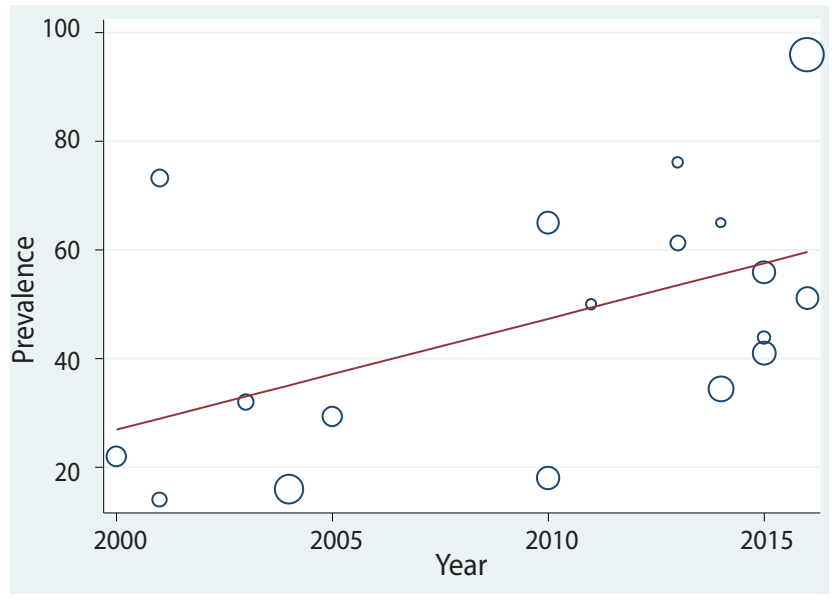

Figure 3. Prevalence of depression among Iranian breast cancer, showing an increase in prevalence from 2000 to 2018.

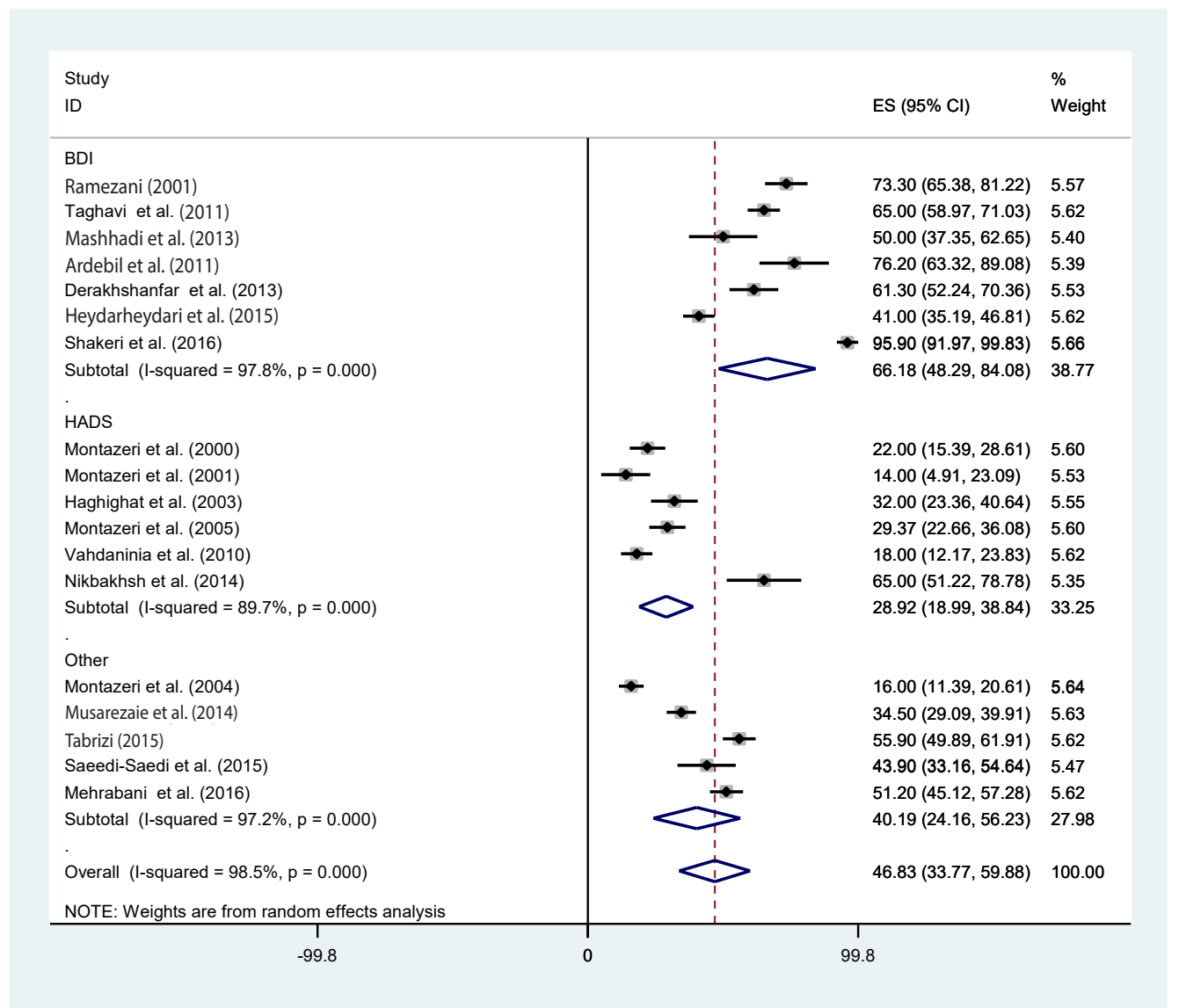

Figure 2. Forest plot of all 18 studies included in the meta-analysis based on the depression assessment tool used. ES, effect size (prevalence); $\mathrm{Cl}$, confidence interval; BDI, Beck Depression Inventory; HADS, Hospital Anxiety and Depression Scale. 


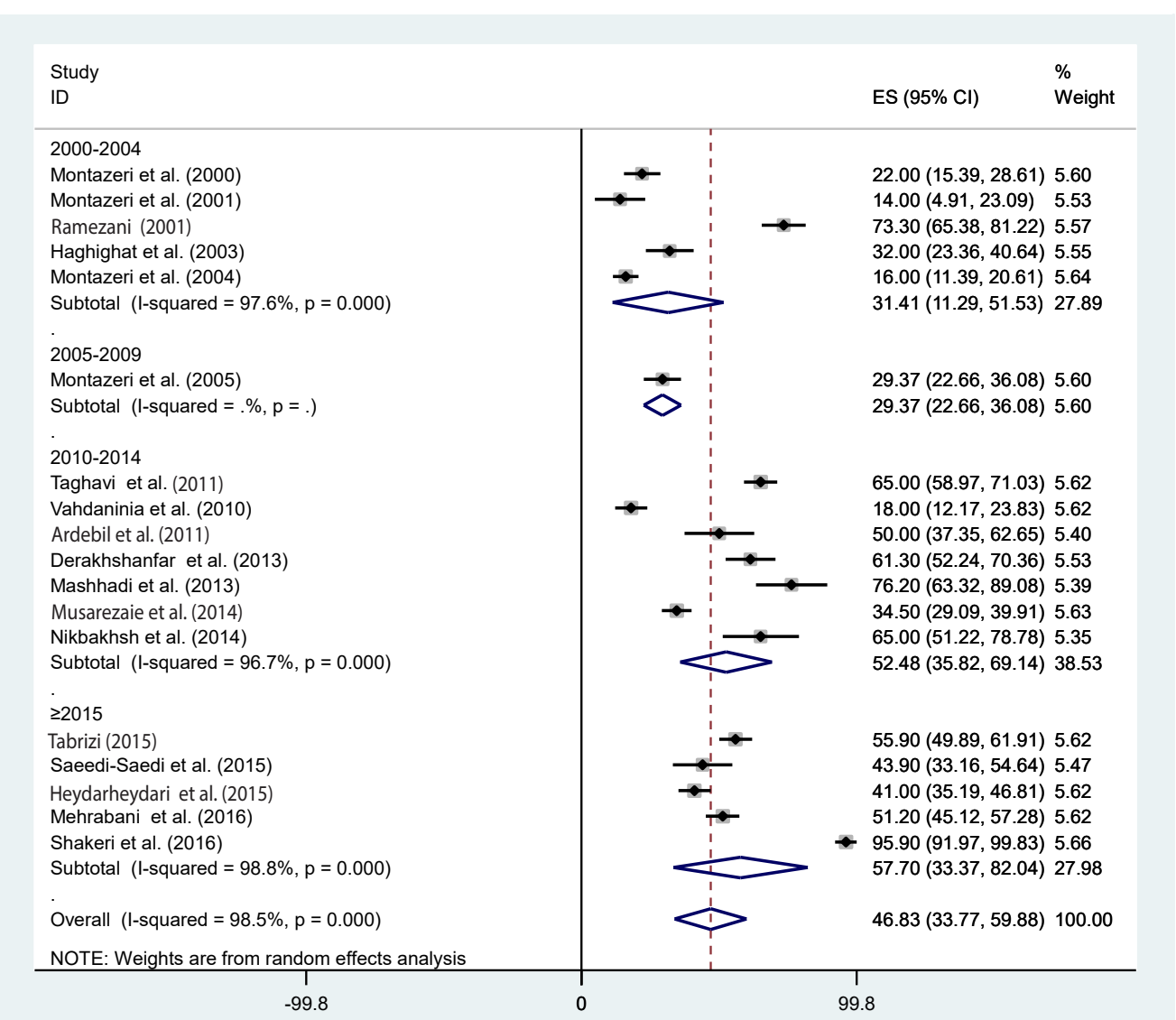

Figure 4. Prevalence of depression among Iranian breast cancer patients based on the year of publication. ES, effect size (prevalence); $\mathrm{Cl}$, confidence interval.

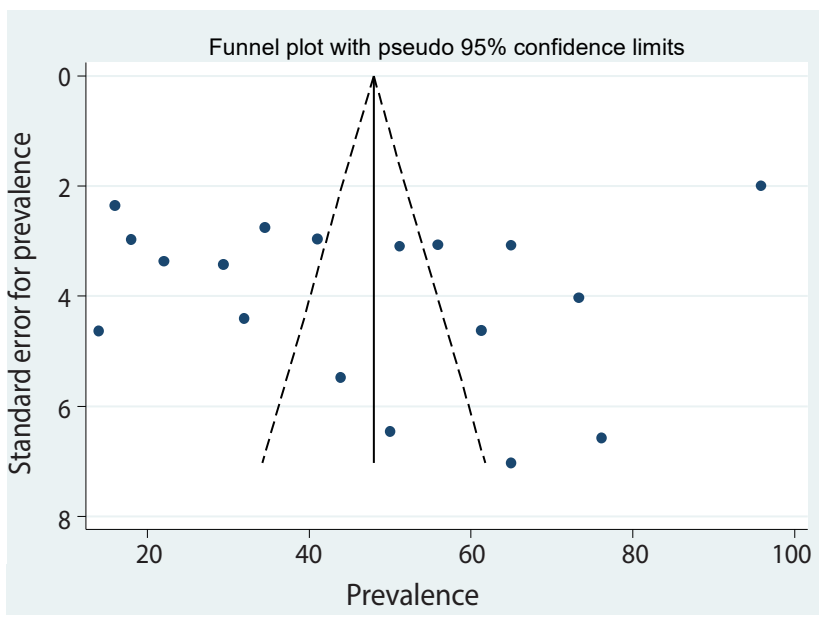

Figure 5. Funnel plot of the studies included in the meta-analysis.

shown in Table 3. The results of the subgroup analysis by study design and tool for depression assessment are shown in Figures 2 and 6 , respectively.
Table 3. Sensitivity analysis of studies included in the meta-analysis

\begin{tabular}{lccc}
\hline \multirow{2}{*}{ Study } & & \multicolumn{2}{c}{$95 \%$ Cl } \\
\cline { 3 - 4 } & Estimate & LL & UL \\
\hline Shakeri et al., 2016 [30] & 43.73 & 34.32 & 53.14 \\
Mehrabani et al., 2016 [32] & 46.57 & 32.63 & 60.13 \\
Heydarheydari et al., 2015 [23] & 47.18 & 33.22 & 61.13 \\
Tabrizi, 2015 [4] & 46.29 & 32.38 & 60.20 \\
Saeedi-Saedi et al., 2015 [2] & 47.00 & 33.40 & 60.59 \\
Musarezaie et al., 2014 [27] & 47.56 & 33.65 & 61.48 \\
Nikbakhsh et al., 2014 [28] & 45.80 & 32.31 & 59.28 \\
Derakhshanfar et al., 2013 [25] & 45.98 & 32.37 & 59.59 \\
Ardebil et al., 2011 [21] & 46.64 & 33.10 & 60.19 \\
Mashhadi et al., 2013 [24] & 45.15 & 31.72 & 58.58 \\
Taghavi et al., 2011 [31] & 45.75 & 31.99 & 59.50 \\
Vahdaninia et al., 2010 [36] & 48.54 & 35.22 & 61.85 \\
Montazeri et al., 2005 [26] & 47.86 & 34.19 & 61.53 \\
Montazeri et al., 2004 [35] & 48.66 & 35.70 & 61.62 \\
Haghighat et al., 2003 [22] & 47.70 & 34.09 & 61.30 \\
Ramezani, 2001 [29] & 45.26 & 31.77 & 58.76 \\
Montazeri et al., 2001 [34] & 48.74 & 35.41 & 62.08 \\
Montazeri et al., 2000 [33] & 48.30 & 34.81 & 61.79 \\
Combined & 46.82 & 33.77 & 62.08 \\
\hline
\end{tabular}

$\mathrm{Cl}$, confidence interval; LL, lower limit; UL, upper limit. 




Figure 6. Forest plot of all studies based on study design. ES, effect size (prevalence); $\mathrm{Cl}$, confidence interval.

\section{Estimate of pooled prevalence in the subgroup analysis based on the result of meta-regression}

The pooled depression prevalence measured by the BDI, HADS, and other methods was $66.18 \%$ (95\% CI, 48.29 to 84.08 ), $28.92 \%$ (95\% CI, 18.99 to 38.84 ), and $40.19 \%$ (95\% CI, 24.16 to 56.23), respectively (Figure 2). Moreover, the prevalence of depression reported by cross-sectional and prospective studies was $55.28 \%$ (95\% CI, 42.48 to 68.08 ) and $17.54 \%$ (95\% CI, 14.55 to 20.54), respectively (Figure 6). The depression prevalence based on year of publication was $22.00 \%$ (95\% CI, 15.39 to 28.61) and $73.61 \%$ (95\% CI, 29.81 to 100.00 ) in 2000 and 2016, respectively (Figure 4).

\section{DISCUSSION}

Depression is a common condition among BC patients, but it is often unrecognized and therefore untreated. The condition intensifies physical symptoms, resulting in additive functional impairment and poor adherence to treatment. As a result, depression in $\mathrm{BC}$ patients is responsible for significant deterioration in QoL [9].

The current systematic review and meta-analysis was conducted to investigate the prevalence of depression among Iranian women diagnosed with BC. The high prevalence (46.8\%) of depression observed in this study among BC survivors is worrying, and it calls for immediate attention. We found that the lowest and highest reported prevalence rates were $14.0 \%$ in Tehran [34] and 95.9\% in Kermanshah [30], respectively. Our results suggest that the prevalence of depression among Iranian BC patients is higher than that of postpartum depression in Iran, depression among Iranian infertile couples, or depression among Iranian adolescents [37-39]. According to the results of a systematic review, the global prevalence of depression among women with BC ranged from $1 \%$ in London to 56\% in USA [40]. Furthermore, another study reported that the prevalence of depression among Asian women with BC was between $12.5 \%$ and $31.0 \%$ [40]. Our study showed that the prevalence of depression among Iranian women with BC is higher than rates that have been reported globally [41,42]. A study conducted in Turkey reported the prevalence of depression among BC patients ranged from $27.7 \%$ (moderate depression) to $19.5 \%$ (major depression) [41]. Similarly, a previous systematic review on prevalence of depression and anxiety after BC treatment showed a lower rate of depression among women with BC (between 9.4\% and $66.1 \%$ ) [42]. The observed differences in the prevalence of depression among women from different countries are likely due to differences in cultural, behavioral, and demographic characteristics, including the economic status of the population [43-45], age (Iranian patients are younger) [36], social support [46], education [47], and marital status [48]. In addition, discrepancies in cancer 
stage and illness duration, differences in the methods used to measure depression, and variation in sample size and methods of sampling may have led to different prevalence rates.

The results of this study showed that the prevalence of depression increased from 2000 to 2017; the prevalence of depression in 2000-2004 years was 31.4\%, and after 2015 it was around 57.7\%. Many factors may have an effect on the prevalence of depression among $\mathrm{BC}$ patients, including increases in the prevalence and incidence of $\mathrm{BC}$, increases in the survival rate of patients, improvements in diagnostic methods, and the launch of a cancer registration system.

It seems that in Iranian society, for cultural reasons, women are placed under additional pressure following the diagnosis and treatment of BC. As a result, while suffering from physical complications of the disease and its treatment, $\mathrm{BC}$ patients suffer from stress and emotional problems [49]. The higher prevalence of depression among BC survivors in Iran can be explained as the result of lack of support from society and patients' families. As BC and its treatment strongly influence the daily activity and sexual performance of survivors at sexually active ages, younger patients are especially prone to depression. It has been suggested that the risk of sexual dysfunction is higher among younger women due to the dependence of their sexual desire on their body image and a reduction in their self-esteem after BC treatment (i.e., mastectomy) [50]. Furthermore, psychological problems and sexual dysfunction after $\mathrm{BC}$ treatment have been reported in the majority of patients [51,52]. For example, a study of Iranian patients suggested that after treatment (i.e., mastectomy), women had negative feelings about their bodies [49]. It has also been suggested that when Iranian women with $\mathrm{BC}$ are about to engage in sexual intercourse, they face anxiety, shame, and negative feelings about their bodies [49].

The opinion of relatives, especially the husband's relatives, about the patient is another source of stress. Emotional support from their husbands is therefore fundamentally important for patients during the treatment of BC. It has been shown that society's and relatives' attitude toward women who have lost their feminine organs have significant effects on their feelings, potentially resulting in depression and anxiety [52]. This issue is mainly due to the cultural context of society, and to some extent the religious beliefs of a community toward women's responsibility as wives to build a satisfactory sexual relationship with their husbands. When facing challenges in fulfilling these expectations, patients may feel incompetent and sense that they are subject to tremendous pressure $[49,51]$. These factors, along with the physical complications of the disease and its treatments, may be unbearable to patients who need social and emotional support. This lack of support makes patients prone to depression and reduces their QoL [49].

Another important factor that may have a significant effect on depression among patients with $\mathrm{BC}$ is the late diagnosis of $\mathrm{BC}$, as around $70 \%$ of Iranian patients with BC are diagnosed at an advanced stage [3], which is again due to low levels of knowledge and access to medical services and cultural barriers [53]. As a consequence of late diagnosis, patients face more aggressive tu- mors, require more aggressive treatment (i.e., mastectomy), and have a poorer prognosis. It has been suggested that patients who receive aggressive treatment or surgery are more prone to psychological problems, including depression, as breasts are a symbol of femininity and their partial or total removal can cause irreversible damage to patients' feelings and emotional state [54].

Along with the previously discussed issues, the observed difference in the prevalence of depression reported by previous studies can also be explained in terms of the studies' design. In this review study, the prevalence of depression reported in cross-sectional studies was much higher (55.3\%) than that reported in prospective studies (17.5\%). In line with this result, another review article reported that the prevalence of depression in crosssectional studies was higher than in prospective studies [40]. A possible reason for this is that cross-sectional studies usually include both new and old cases of depression. In addition, the tools used by the studies included in this systematic review were rather heterogeneous. In studies that used the BDI scale, the prevalence of depression among BC patient was much higher (66.2\%) than that reported in studies using the HADS (28.9\%), and studies utilizing other scales (DSM-IV, DASS-42, CES-D, and the DT) showed an intermediate prevalence of depression (around 40\%). The BDI seems to be a valid screening tool for depression in advanced cancer patients [55], although the HADS is also a useful screening tool for depression in cancer patients [56]. However, it seems that the differences in the results of the BDI and HADS should be acknowledged, meaning that results should be compared with caution. A possible reason for discrepancies in the results of different depression scales was provided by Cusin et al. [57]. They stated that only the HADS is suitable for measuring depression in those with a serious medical condition; other measures might overestimate the scale of depression, since they do not exclude somatic factors [57]. However, there is no consensus on this issue, as Hann et al. [58] evaluated the CES-D in BC patients and concluded that the scale was a valid and reliable measure for diagnosing depression in BC survivors. In a similar vein, Osborne et al. [59] investigated the validity of the HADS in BC survivors, and only found minor psychometric problems.

The results of the current study revealed a high prevalence of depression among women with BC. Suffering from depression can exacerbate the prognosis of the disease and reduce patients' survival [60]. According to the results of this study, there was no relationship between prevalence of depression and the age of women with BC. Our finding is consistent with the results of a study by Vin-Raviv et al. [61] in this regard. However, other studies have revealed a direct association between the prevalence of depression and age [62].

The present study has some limitations that must be pointed out. Firstly, data on some important factors, including the stage of $\mathrm{BC}$ and the years since diagnosis, were not available, making it impossible to conduct a subgroup analysis of whether the prevalence of depression differed according to those factors. Secondly, Iran is a country with considerable ethnic diversity, which may 
affect the prevalence of depression in different areas of the country. However, it was not possible for us to determine the possible role of ethnicity in these findings. In contrast, strengths of this study include the fact that national and international databases were searched in both Farsi (Persian) and English to obtain comprehensive results, and that we excluded studies on $\mathrm{BC}$ recurrence because this may be a source of bias.

\section{CONCLUSION}

The results of this systematic review revealed a remarkably high prevalence of depression among women with BC. Early diagnosis, social support, and emotional support from family members can help in the management of depression, with positive impacts on QoL. These results should be carefully considered by physicians, healthcare providers, and the patient's family when a woman is diagnosed with BC.

\section{SUPPLEMENTARY MATERIALS}

Supplementary material is available at http://www.e-epih.org/.

\section{CONFLICT OF INTEREST}

The authors have no conflicts of interest to declare for this study.

\section{ACKNOWLEDGEMENTS}

This study was funded by a grant of the Tehran University of Medical Sciences (No. 36080).

\section{AUTHOR CONTRIBUTIONS}

Conceptualization: MD, MF. Data curation: HAG, SMK, MD. Formal analysis: HAG, RP. Funding acquisition: HAG. Methodology: RG, MD. Project administration: MD, HAG, RG. Visualization: MF, RG, MD. Writing - orginal draft: HAG, HM, SMK, RP. Writing - review \& editing: MD, RG, MF.

\section{ORCID}

Hasan Ahmadi Gharaei: https://orcid.org/0000-0002-7485-1612; Mostafa Dianatinasab: https://orcid.org/0000-0002-0185-5807; Seyyed Mostafa Kouhestani: https://orcid.org/0000-0002-0122671X; Mohammad Fararouei: https://orcid.org/0000-0001-9362328X; Hossein Moameri: https://orcid.org/0000-0003-0505-4473; Reza Pakzad: https://orcid.org/0000-0001-8133-3664; Reza Ghaiasvand: $h$ ttps://orcid.org/0000-0002-5466-7308

\section{REFERENCES}

1. Bray F, Ferlay J, Soerjomataram I, Siegel RL, Torre LA, Jemal A. Global cancer statistics 2018: GLOBOCAN estimates of incidence and mortality worldwide for 36 cancers in 185 countries. CA Cancer J Clin 2018;68:394-424.

2. Saeedi-Saedi H, Shahidsales S, Koochak-Pour M, Sabahi E, Moridi I. Evaluation of emotional distress in breast cancer patients. Iran J Cancer Prev 2015;8:36-41.

3. Dianatinasab M, Mohammadianpanah M, Daneshi N, Zare-Bandamiri M, Rezaeianzadeh A, Fararouei M. Socioeconomic factors, health behavior, and late-stage diagnosis of breast cancer: considering the impact of delay in diagnosis. Clin Breast Cancer 2018;18:239-245.

4. Tabrizi FM. Health promoting behavior and influencing factors in Iranian breast cancer survivors. Asian Pac J Cancer Prev 2015; 16:1729-1736.

5. Cvetković J, Nenadović M. Depression in breast cancer patients. Psychiatry Res 2016;240:343-347.

6. Burgess C, Cornelius V, Love S, Graham J, Richards M, Ramirez A. Depression and anxiety in women with early breast cancer: five year observational cohort study. BMJ 2005;330:702.

7. Ho SS, So WK, Leung DY, Lai ET, Chan CW. Anxiety, depression and quality of life in Chinese women with breast cancer during and after treatment: a comparative evaluation. Eur J Oncol Nurs 2013;17:877-882.

8. DeSantis CE, Ma J, Goding Sauer A, Newman LA, Jemal A. Breast cancer statistics, 2017, racial disparity in mortality by state. CA Cancer J Clin 2017;67:439-448.

9. Fann JR, Thomas-Rich AM, Katon WJ, Cowley D, Pepping M, McGregor BA, et al. Major depression after breast cancer: a review of epidemiology and treatment. Gen Hosp Psychiatry 2008; 30:112-126.

10. Walker J, Hansen CH, Martin P, Symeonides S, Ramessur R, Murray $\mathrm{G}$, et al. Prevalence, associations, and adequacy of treatment of major depression in patients with cancer: a cross-sectional analysis of routinely collected clinical data. Lancet Psychiatry 2014;1:343-350.

11. Brandão T, Schulz MS, Matos PM. Psychological adjustment after breast cancer: a systematic review of longitudinal studies. Psychooncology 2017;26:917-926.

12. Kashani F, Babaee S, Bahrami M, Valiani M. The effects of relaxation on reducing depression, anxiety and stress in women who underwent mastectomy for breast cancer. Iran J Nurs Midwifery Res 2012;17:30-33.

13. Baniasadi H, kashani FK, Jamshidifar Z. Effectiveness of mindfulness training on reduction of distress of patients infected by breast cancer. Procedia Soc Behav Sci 2014;114:944-948.

14. Hu Y, Liu T, Li F. Association between dyadic interventions and outcomes in cancer patients: a meta-analysis. Support Care Cancer 2019;27:745-761.

15. McInnes MD, Moher D, Thombs BD, McGrath TA, Bossuyt PM; the PRISMA-DTA Group, et al. Preferred reporting items for a systematic review and meta-analysis of diagnostic test accuracy studies: the PRISMA-DTA statement. JAMA 2018;319:388-396.

16. Loney PL, Chambers LW, Bennett KJ, Roberts JG, Stratford PW. Critical appraisal of the health research literature: prevalence or 
incidence of a health problem. Chronic Dis Can 1998;19:170-176.

17. Higgins JP, Thompson SG, Deeks JJ, Altman DG. Measuring inconsistency in meta-analyses. BMJ 2003;327:557-560.

18. Egger M, Davey Smith G, Schneider M, Minder C. Bias in metaanalysis detected by a simple, graphical test. BMJ 1997;315:629634.

19. Sterne JA, Egger M. Funnel plots for detecting bias in meta-analysis: guidelines on choice of axis. J Clin Epidemiol 2001;54:10461055.

20. Harris RJ, Bradburn M, Deeks J, Harbord RM, Altman D, Steichen T, et al. METAN: Stata module for fixed and random effects meta-analysis; 2007 [cited 2019 May 1]. Available from: https://research-information.bristol.ac.uk/en/publications/metan-statamodule-for-fixed-and-random-effects-metaanalysis(f586792bcd5b-468d-95e8-46dbb62eba96).html.

21. Ardebil MD, Bouzari Z, Shenas MH, Zeinalzadeh M, Barat S. Depression and health related quality of life in breast cancer patients. Acad J Cancer Res 2011;4:43-46.

22. Haghighat S, Akbari ME, Holakouei K, Rahimi A, Montazeri A. Factors predicting fatigue in breast cancer patients. Support Care Cancer 2003;11:533-538.

23. Heydarheydari S, Khalili M, Sadeghi S. The relationship between anxiety and depression with breast cancer screening in women referring to the mammography clinics in Kermanshah, 2013-2014. J Clin Res Paramed Sci 2015;4:231-237 (Persian).

24. Mashhadi MA, Shakiba M, Zakeri Z. Evaluation of depression in patients with cancer in South of Iran (Zahedan). Iran J Cancer Prev 2013;6:12-16.

25. Derakhshanfar A, Niayesh A, Abbasi M, Ghalaeeha A, Shojaee M. Frequency of depression in breast cancer patients: a study in Farshchian and Besat Hospitals of Hamedan during 2007-8. Iran J Surg 2013;21:68-74 (Persian).

26. Montazeri A, Sajadian A, Ebrahimi M, Akbari ME. Depression and the use of complementary medicine among breast cancer patients. Support Care Cancer 2005;13:339-342.

27. Musarezaie A, Momeni-GhaleGhasemi T, Gorji M. Survey the anxiety and depression among breast cancer patients referred to the specialized Isfahan hospital of cancer. Iran J Health Syst Res 2014;10:39-48 (Persian).

28. Nikbakhsh N, Moudi S, Abbasian S, Khafri S. Prevalence of depression and anxiety among cancer patients. Caspian J Intern Med 2014;5:167-170.

29. Ramezani T. Degree of depression and the need for counseling among women with breast cancer in Kerman chemotherapeutic centers. Iran J Psychiatry Clin Psychol 2001;6:70-80 (Persian).

30. Shakeri J, Golshani S, Jalilian E, Farnia V, Nooripour R, Alikhani $\mathrm{M}$, et al. Studying the amount of depression and its role in predicting the quality of life of women with breast cancer. Asian Pac J Cancer Prev 2016;17:643-646.

31. Taghavi M, Kalafi E, Talei A, Dehbozorgi G, Taghavi SM. Investigating the relation of depression and religious coping and social support in women with breast cancer. J Isfahan Med Sch 2011;28: 901-908 (Persian).
32. Mehrabani F, Barati F, Ramezanzade Tabriz E, Bakaeian M, Gholami Chaboki B. Unpleasant emotions (stress, anxiety and depression) and it is relationship with parental bonding and disease and demographic characteristics in patients with breast cancer. Iran Q J Breast Dis 2016;9:42-52 (Persian).

33. Montazeri A, Harirchi I, Vahdani M, Khaleghi F, Jarvandi S, Ebrahimi M, et al. Anxiety and depression in Iranian breast cancer patients before and after diagnosis. Eur J Cancer Care (Engl) 2000; 9:151-157.

34. Montazeri A, Jarvandi S, Haghighat S, Vahdani M, Sajadian A, Ebrahimi M, et al. Anxiety and depression in breast cancer patients before and after participation in a cancer support group. Patient Educ Couns 2001;45:195-198.

35. Montazeri A, Jarvandi S, Ebrahimi M, Haghighat S, Ansari M. The role of depression in the development of breast cancer: analysis of registry data from a single institute. Asian Pac J Cancer Prev 2004;5:316-319.

36. Vahdaninia M, Omidvari S, Montazeri A. What do predict anxiety and depression in breast cancer patients? A follow-up study. Soc Psychiatry Psychiatr Epidemiol 2010;45:355-361.

37. Veisani Y, Delpisheh A, Sayehmiri K, Rezaeian S. Trends of postpartum depression in Iran: a systematic review and meta-analysis. Depress Res Treat 2013;2013:291029.

38. Masoumi SZ, Poorolajal J, Keramat A, Moosavi SA. Prevalence of depression among infertile couples in Iran: a meta-analysis study. Iran J Public Health 2013;42:458-466.

39. Veisani Y, Delpisheh A, Sayehmiri K, Rezaeian S. Trends of postpartum depression in Iran: a systematic review and meta-analysis. Depress Res Treat 2013;2013:291029.

40. Zainal NZ, Nik-Jaafar NR, Baharudin A, Sabki ZA, Ng CG. Prevalence of depression in breast cancer survivors: a systematic review of observational studies. Asian Pac J Cancer Prev 2013;14: 2649-2656.

41. Bener A, Alsulaiman R, Doodson L, Agathangelou T. Depression, hopelessness and social support among breast cancer patients: in highly endogamous population. Asian Pac J Cancer Prev 2017;18:1889-1896.

42. Maass SW, Roorda C, Berendsen AJ, Verhaak PF, de Bock GH. The prevalence of long-term symptoms of depression and anxiety after breast cancer treatment: a systematic review. Maturitas 2015;82:100-108.

43. Chen X, Zheng Y, Zheng W, Gu K, Chen Z, Lu W, et al. Prevalence of depression and its related factors among Chinese women with breast cancer. Acta Oncol 2009;48:1128-1136.

44. Eversley R, Estrin D, Dibble S, Wardlaw L, Pedrosa M, Favila-Penney W. Post-treatment symptoms among ethnic minority breast cancer survivors. Oncol Nurs Forum 2005;32:250-256.

45. Morrill EF, Brewer NT, O’Neill SC, Lillie SE, Dees EC, Carey LA, et al. The interaction of post-traumatic growth and post-traumatic stress symptoms in predicting depressive symptoms and quality of life. Psychooncology 2008;17:948-953.

46. Den Oudsten BL, Van Heck GL, Van der Steeg AF, Roukema JA, De Vries J. Predictors of depressive symptoms 12 months after 
surgical treatment of early-stage breast cancer. Psychooncology 2009;18:1230-1237.

47. Broeckel JA, Jacobsen PB, Balducci L, Horton J, Lyman GH. Quality of life after adjuvant chemotherapy for breast cancer. Breast Cancer Res Treat 2000;62:141-150.

48. Pumo V, Milone G, Iacono M, Giuliano SR, Di Mari A, Lopiano C, et al. Psychological and sexual disorders in long-term breast cancer survivors. Cancer Manag Res 2012;4:61-65.

49. Fouladi N, Pourfarzi F, Dolattorkpour N, Alimohammadi S, Mehrara E. Sexual life after mastectomy in breast cancer survivors: a qualitative study. Psychooncology 2018;27:434-441.

50. Pinto AC. Sexuality and breast cancer: prime time for young patients. J Thorac Dis 2013;5 Suppl 1:S81-S86.

51. Harirchi I, Montazeri A, Zamani Bidokhti F, Mamishi N, Zendehdel K. Sexual function in breast cancer patients: a prospective study from Iran. J Exp Clin Cancer Res 2012;31:20.

52. Chen CL, Liao MN, Chen SC, Chan PL, Chen SC. Body image and its predictors in breast cancer patients receiving surgery. Cancer Nurs 2012;35:E10-E16.

53. Montazeri A, Ebrahimi M, Mehrdad N, Ansari M, Sajadian A. Delayed presentation in breast cancer: a study in Iranian women. BMC Womens Health 2003;3:4.

54. Fisher C, O'Connor M. "Motherhood" in the context of living with breast cancer. Cancer Nurs 2012;35:157-163.

55. Warmenhoven F, van Rijswijk E, Engels Y, Kan C, Prins J, van Weel C, et al. The Beck Depression Inventory (BDI-II) and a single screening question as screening tools for depressive disorder in Dutch advanced cancer patients. Support Care Cancer 2012; 20:319-324.

56. Wang GL, Hsu SH, Feng AC, Chiu CY, Shen JF, Lin YJ, et al. The HADS and the DT for screening psychosocial distress of cancer patients in Taiwan. Psychooncology 2011;20:639-646.

57. Cusin C, Yang H, Yeung A, Fava M. Rating scales for depression. In: Baer L, Blais MA, editors. Handbook of clinical rating scales and assessment in psychiatry and mental health. Totowa: Humana Press; 2010, p. 7-35.

58. Hann D, Winter K, Jacobsen P. Measurement of depressive symptoms in cancer patients: evaluation of the Center for Epidemiological Studies Depression Scale (CES-D). J Psychosom Res 1999; 46:437-443.

59. Osborne RH, Elsworth GR, Sprangers MA, Oort FJ, Hopper JL. The value of the Hospital Anxiety and Depression Scale (HADS) for comparing women with early onset breast cancer with population-based reference women. Qual Life Res 2004;13:191-206.

60. Noorbala AA, Damari B, Riazi IS. Evaluation of mental disorders incidence trend in Iran. Daneshvar Med 2014;21:1-10 (Persian).

61. Vin-Raviv N, Akinyemiju TF, Galea S, Bovbjerg DH. Depression and anxiety disorders among hospitalized women with breast cancer. PLoS One 2015;10:e0129169.

62. Mehnert A, Koch U. Psychological comorbidity and health-related quality of life and its association with awareness, utilization, and need for psychosocial support in a cancer register-based sample of long-term breast cancer survivors. J Psychosom Res 2008; 64:383-391. 